\title{
Caín, Abel y Francia 98
}

El mundo entero se ve sacudido de nuevo por la fiebre de la Copa Mundial de Fútbol, iy vaya si es fiebre! En las oficinas y centros educativos se vuelve problemático el desarrollo de las actividades dado el poco interés por nada que no sea Francia, Ronaldo o el buen estado del televisor (no cuesta mucho imaginar lo que le pasaría a un ladrón de televisores en esta época: sólo los suicidios, ante la desaparición de los aparatos, serían más grotescos que los linchamientos y torturas sistemáticas a los que sería sometido). Los ánimos se encuentran exaltados, y los jefes, catedráticos y maestros de escuela nos las ingeniamos con métodos coercitivos para evitar el éxodo inmisericorde de nuestros respectivos lugares de trabajo.

Por otro lado, es paradójico que mientras se aboga en los estadios galos por el juego limpio, en la "guanaxia" vale todo con tal de apropiarse esta realidad y sacar las respectivas ganancias. Los televidentes y fanáticos del deporte universal no sólo tenemos que ver los partidos adivinando el nombre de los jugadores, gracias a sendos cartelitos de los anunciantes que compiten en rapidez con los delanteros, sino que tenemos que tragarnos a unos comentaristas que a su folklorismo añaden una buena dosis de agresividad y nos regalan los esquemas más antideportivos que es posible encontrar. Sí, adivinaron, nos referimos a la transmisión exclusiva del Canal Cuatro' .

Valga esta introducción como excusa para reflexionar sobre lo que puede hacer el deporte en una sociedad sacudida por la violencia, como la nuestra, y para señalar qué actitudes no abonan para nada a la solución de tal problema. Para ello me permitiré utilizar algunas ideas del etólogo Konrad Lorenz, estudioso del comportamiento de los animales y del hombre. Este sostiene, luego de un largo pero muy interesante análisis sobre el papel de la agresividad intraespecifica en los animales ${ }^{2}$, que tal fenómeno, la agresividad, es de fundamental importancia para la supervivencia de la especie y para su desarrollo armónico y equilibrado. Y el hombre no es la excepción. Ahora bien, la agresividad para con los miembros de la misma especie tiene ciertos límites, determinados en los animales por el código genético: hay instintos que restringen la agresividad; se trata de un contrapeso biológico. En el hombre también existe la agresividad intraespecífica, no hace falta insistir en ello. Pero, y aquí se encuentra lo que podría ser polémico, esta agresividad es institiva. En este punto, nuestra filiación animal queda evidenciada, pero es justo en los mecanismos restrictivos de la agresividad donde aparece la diferencia con aquéllos: el hombre no posee tales instintos que sirvan de contrapeso al instinto agresivo. Es aquí donde hace su aparición la moral.

Ahora bien, Lorenz es perfectamente consciente de que la restricción animal nunca es total, ya que la agresividad cumple con funciones verdaderamente positivas, tales como la selección de aquellos individuos más fuertes para la procreación. Pero no sólo eso, sino que él ve en el instinto agre-

1. Cfr., El Diario de Hoy, 10 de junio de 1998, pp. 120-121.

2. Cfr. Lorenz, K. Sobre la agresión: el pretendido mal, México: Siglo XXI editores, 1978. 
sivo el punto de partida, el posibilitante de lo que podría llamarse el "vínculo afectivo" existente en muchas especies animales. La necesidad de controlar los instintos agresivos da origen a las "luchas ritualizadas", las cuales reorientan sin eliminar del todo lo agresivo hacia la obtención de un comportamiento útil: el vínculo entre dos individuos. Este vínculo es lo que entre humanos llamamos amor, y parece ser que se origina, según Lorenz, en los impulsos agresivos. No hará falta mucha imaginación para ver la mutua imbricación entre amor y odio que existe entre las parejas humanas, pero por ahora nos interesa destacar otra cosa: el papel que juegan las luchas ritualizadas en la reorientación positiva de la agresión.

En el fondo de estos planteamientos encontramos dos ideas interesantes: la tendencia evolutiva ha sido la de la reorientación de la agresión más que la eliminación de la misma, y parece que entre humanos no es la excepción; por otro lado, esta reorientación de la agresividad es positiva. Es asi que, para Lorenz, las luchas ritualizadas pasarán a cumplir con "la difícil misión de evitar la muerte del antagonista sin acabar al mismo tiempo con la combatividad, tan importante para la conservación de la especie. Todas las normas de 'lucha limpia' de origen cultural, desde la caballería andante hasta los acuerdos de Ginebra, han tenido una función análoga a la de los combates filogenéticamente ritualizados de los animales"3.

Pero, ¿qué tiene que ver esto con el Mundial? ¿Es que puede considerarse al fútbol como una "lucha ritualizada"? Pues sí, justamente aquí entra nuestra reflexión más adecuada a la realidad de nuestro país, a su pasión por el deporte y la dura realidad de violencia que le aqueja.

Nosotros sostenemos que el deporte puede servir perfectamente para la canalización positiva de la agresividad presente en la sociedad salvadoreñ. Hasta aquí no hay nada nuevo, pero la cosa cambia y se complejiza al reflexionar más a fondo en lo que entenderemos precisamente por deporte. Recurrimos de nuevo a Lorenz:

"Es el deporte una forma de lucha ritualizada especial, producto de la vida cultural humana. Procede de luchas serias, pero fuertemente ritualizadas. A la manera de los combates codificados, de los 'duelos por el honor', de origen filogenético, impide los efectos de la agresión perjudiciales para la sociedad y al mismo tiempo mantiene incólumes las funciones conservadoras de la especie. Pero además, esta forma culturalmente ritualizada de combate cumple la tarea incomparablemente importante de enseñar al hombre a dominar de modo consciente y responsable sus reacciones instintivas en el combate. La caballerosidad o 'limpieza' del juego deportivo, que se ha de conservar en los momentos más excitantes y desencadenadores de agresión, es una importante conquista cultural de la humanidad. Además, el deporte tiene un carácter benéfico porque hace posible la competencia verdaderamente entusiasta entre dos comunidades supraindividuales. No solamente abre una oportuna válvula de seguridad a la agresión acumulada en la forma de sus pautas de comportamiento más toscas, individuales y egoístas, sino que permite el desahogo cumplido de su forma especial colectiva más altamente diferenciada. La lucha por la jerarquía superior dentro del grupo, la ruda acometida común por un objetivo apasionante, el arrostrar animosamente grandes peligros, el socorro mutuo con olvido de la vida propia, etc., etc., son pautas de comportamiento que en la prehistoria de la humanidad tenían un gran valor selectivo"4.

El punto clave en estas reflexiones es la afirmación de que el juego limpio es una de las más importantes conquistas de la humanidad. ¿Qué es juego limpio? ¿Lo que en la vida cotidiana se nos presenta como deporte está siempre ligado al juego limpio? ¿Cómo se encuentra la relación deportejuego limpio en El Salvador? Estas preguntas guían desde ahora nuestras reflexiones.

¿En qué consiste el juego limpio? En primer lugar diremos que éste implica el reconocimiento del otro en tanto contrincante, o sea, la dimensión de lucha que tiene el juego. He aquí el carácter del juego como competencia: el más competente alcanza la victoria. En segundo lugar, el reconocimiento de que la agresividad no debe dañar o eliminar al contrincante, o sea, la dimensión de rito

3. Jbid., p. 315.

4. Ibid., pp. 315-316. 
en el juego. Estamos hablando del carácter del juego como lugar de encuentro social: el ganador no mata al perdedor. Finalmente, la constatación de la necesidad de reglas, es decir, la dimensión normativa del juego. He aquí el carácter moral de la competencia deportiva: jugadores y espectadores reconocen el valor de las reglas. Ahora, una vez tenemos claro esto del juego limpio, es preciso ver en qué medida está o no presente en los eventos deportivos regulares. Por razones de espacio, orden y situs cultural nos limitaremos al fútbol.

Eso que llamamos fútbol no puede ser reducido actualmente al sólo hecho de veintidós jugadores y tres árbitros disputando un emocionante juego alrededor de la ingente tarea de meter un balón en una portería -o evitarlo. Fútbol, hoy, es también espectáculo, industria, arte, negocio, dinero. Fútbol es identidad nacional, conflicto cultural, foro político y hasta desencadenante de guerras. Por lo tanto, seríamos ingenuos si redujéramos el asunto del juego limpio a la cancha, a las patadas y a los goles con la mano. Nosotros pensamos que el verdedero problema está fuera de la cancha, en los graderíos de los estadios, las calles con hinchas enardecidos por lemas y consignas, los comentaristas deportivos con sus "ingeniosos" comentarios y los empresarios de los medios con su conducta antiliberal y antidemocrática. Veamos cada uno de estos casos y cómo se presentan en nuestra realidad salvadoreña.

Todo salvadoreño sabe los riesgos en que incurre si se introduce en "El Vietnam", el sector "soleado" del Estadio Cuscatlán. A lo mejor, los mismos riesgos son el reclamo para cientos y miles de guanacos que disfrutan más de las guerras con bolsas inmundas y las explosiones de cohetes que con el partido de turno. Lo que debería ser un lugar donde desahogar las energías y disfrutar sanamente es un verdadero campo de batalla. Y si a esto añadimos el que nuestros estadios no gozan de las mínimas condiciones de seguridad y comodidad, parece que es hora de pedir que se nos juegue limpio.

Si nos trasladamos a la calle nos encontramos con un panorama similar. Dejando de lado el caos vehicular que no pocas veces ha provocado en San
Salvador la celebración de una final entre equipos de la primera división, podría decirse que el vandalismo con justificaciones deportivas no ha llegado a nuestra tierra. Pero sí el nacionalismo enfermizo y fanático que se traduce, a veces, en actos vandálicos: recordemos el cierre del Boulevard de los Héroes la noche anterior al partido entre "La selecta" y el equipo jamaiquino, debido a que los guanacos se habían dado a la tarea de hacer ruido y escándalo con tal de que los visitantes no pudieran descansar. Lo más grave es que una emisora radial había emitido la consigna de cometer tal atropello. Vemos claro que esto no es juego limpio.

$Y$ es que son los medios radial y televisivo los que se llevan los honores en cuanto a la exaltación de la agresividad desbocada en este país. El último partido de la selección nacional jugado en México dio pie a un espectáculo bochomoso por parte del comentarista del Canal Cuatro Eugenio Calderón: "así hay que tratarlos cuando lleguen a El Salvador", vociferaba haciendo alusión a los supuestos insultos y ofensas que recibía en el estadio mexicano. ¡Vaya formación en los valores que reciben los miles de niños salvadoreños que podrían faltar a clases pero no se pierden un partido de "La selecta"! Cuando la zancadilla y el revanchismo antideportivo se convierten en lema y consigna, entonces tenemos de nuevo la impresión de que no se nos está jugando limpio.

Pero el caso más dramático de juego sucio es el más reciente, con ocasión del Mundial de fútbol: el interesante fenómeno que mencionábamos arriba de la transmisión exclusiva de los partidos por parte del Canal Cuatro. El descontento en los usuarios de cable y los dueños de establecimientos comerciales no se hizo esperar, y de poco ayuda el que se pretenda justificar el hecho con argumentos del tipo "se hace en otros lugares", "hay que proteger los derechos de autor" y "di no a la piratería"s. Más bien, los argumentos deberían estar dentro del orden de lo que significa democracia, libre mercado y competitividad equitativa de los agentes comerciales. Un ciudadano descontento con las disposiciones del Canal Cuatro y el aparato de justicia que lo ampara expresó de esta forma su frustración:

5. "La medida, si bien resulta un tanto antipática a simple vista, es común en otros países del orbe. Y en algunos de ellos, esto que no es más que pagar 'derechos', se extiende al campo de la música... Al igual que quienes continúan <<irateando〉> cintas de vídeo o casete" (Cienfuegos, Janet. "Pague si quiere ganar", EDH, 10 de junio de 1998, p. 108). 
"No me parece correcto que el Canal Cuatro de Televisión haya conseguido prohibir que las compañías de cable puedan transmitir el Mundial de Francia 98. Yo creía que vivíamos en una época democrática en donde nosotros podiamos escoger nuestros propios gustos. Ahora nos encontramos con que, obligadamente, tenemos que presenciar a los comentaristas del Canal Cuatro para que nos presenten la última Copa Mundial de nuestro siglo. ¿Tanto es el poder monopólico del Canal Cuatro que vamos a tener que aguantarnos a la fuerza? ${ }^{6}$

La sociedad democrática es precisamente aquella en la cual el ciudadano puede hacer valer frente al todo social y los grupos de poder sus preferencias. Libre mercado significa que no nos pueden obligar a consumir este o aquel producto, sino que podemos elegir el que llene mejor nuestras expectativas. Competitividad equitativa es todo lo contrario al monopolio. En suma, los procedimientos mediante los cuales se adjudican los derechos de transmisión no se basan en la consulta ciudadana y la libre competencia de los medios, en el sentido de ofrecer la calidad y eficiencia que permitan determinar a quién le corresponde por su buen desempeño el derecho de transmisión. Por otro lado, las companías de cable no tendrían por qué plegarse a las disposiciones de las televisoras nacionales, ya que de antemano el usuario de sus servicios ha elegido éste y no el servicio nacional, con lo cual es una verdadera estafa el que se le prive por un servicio por el que ha pagado y que es, objetivamente hablando, más eficiente y de mayor calidad.

Nos servirá perfectamente para nuestra conclusión la canción de Ricky Martin que se ha convertido en el lema del Mundial. Esta no sólo nos permite reconocer el carácter de espectáculo que significa la competición sino que, además, nos regala con una idea ejemplificadora de lo que significa "competitividad" para algunos empresarios del deporte, comentaristas deportivos y dueños de los medios de comunicación: "Como Caín y Abel...". Competir es emular no a dos luchadores que buscan ganar limpiamente, respetando las reglas, sino al asesino que, mediante engaños, no sólo vence a su contrincante sino que lo elimina totalmente, en un claro acto de injusticia y violencia fratricida. Esto es lo que vemos y escuchamos frecuentemente en las transmisiones de los encuentros fútbolísticos; los partidos son anunciados mediante la frase "inicio de las hostilidades". Definitivamente que aquí se habla de agresión, pero no la sana, la que respeta unas convenciones y sirve para reconocer al otro en sus diferencias, sino la agresión que busca borrar al otro de la faz de la tierra.

Nosotros no creemos que habría que volver al lema "lo que importa no es ganar sino competir", en el cual se escuda una visión idealista de la competencia deportiva, en tanto no reconoce el factor importante del "deseo de vencer", presente en tanto "lucha ritualizada", y, además, trasluce una descalificación satanizante de la agresividad. El lema debe ser "lo que importa es ganar, pero limpiamente", reconociendo que la competencia deportiva no sólo divierte, no sólo sirve a la catársis de todo lo agresivo, sino que me enfrenta a otros como yo dentro de un marco de reglas que ambos hemos convenido en aceptar. El deporte como instrumento para la paz entre naciones ya era un asunto importante para Lorenz:

"Las competencias entre naciones no solamente son benéficas porque facilitan la abreacción del entusiasmo nacional sino también porque producen otros dos efectos contrarios a la guema: en primer lugar, procuran un conocimiento personal a hombres y mujeres de diferentes naciones y partidos, $y$, en segundo lugar, hacen que se unan para una causa común personas que de otro modo pocas cosas podrían unir. Son dos fuerzas poderosas opuestas a la agresión, y debemos examinar brevemente de qué modo pueden desplegar su benéfica acción y qué otros medios podrían emplearse para aplicarlas a nuestro objetivo"7.

Un requisito indispensable para ser autoridad en asuntos de deportes sería participar de una visión de la competencia deportiva que asuma estas ideas como abono para la paz social. En la medida en que dejamos tal tarea a deportistas indisciplinados y sin el menor sentido de lo que son reglas de competitividad, a empresarios acostumbrados a la zancadilla y a la farsa para mantenerse en sus puestos, a comentaristas deportivos sin la mí-

6. Zepeda, Rogelio. “Cartas al director", EDH, 10 de junio de 1998, p. 24. (Las cursivas son nuestras.)

7. Lorenz, K Sobre la agresión: el pretendido mah op. cit., pp. 316-317. 
nima idea de lo importante que es su trabajo para la transmisión de valores a la juventud, y a empresarios de los medios que ofrecen a toda una nación claras muestras de conducta antideportiva, en esa medida estaremos muy lejos del derecho a participar en competencias deportivas importantes y, lo que es más serio, el deporte no servirá a la solución del problema de violencia que nos desangra. Tal vez, cuando todos los que nos preocupamos por el deporte en este país hagamos prevalecer los valores deportivos sobre los predatorios, entonces y sólo entonces puede que los salvadoreños merezcamos participar en un Mundial. Y puede que de esa manera nuestra participación ayude a construir en nuestra tierra una cultura auténtica, de valores que contribuyan a la vida y a la paz.

Carlos Molina Velásquez
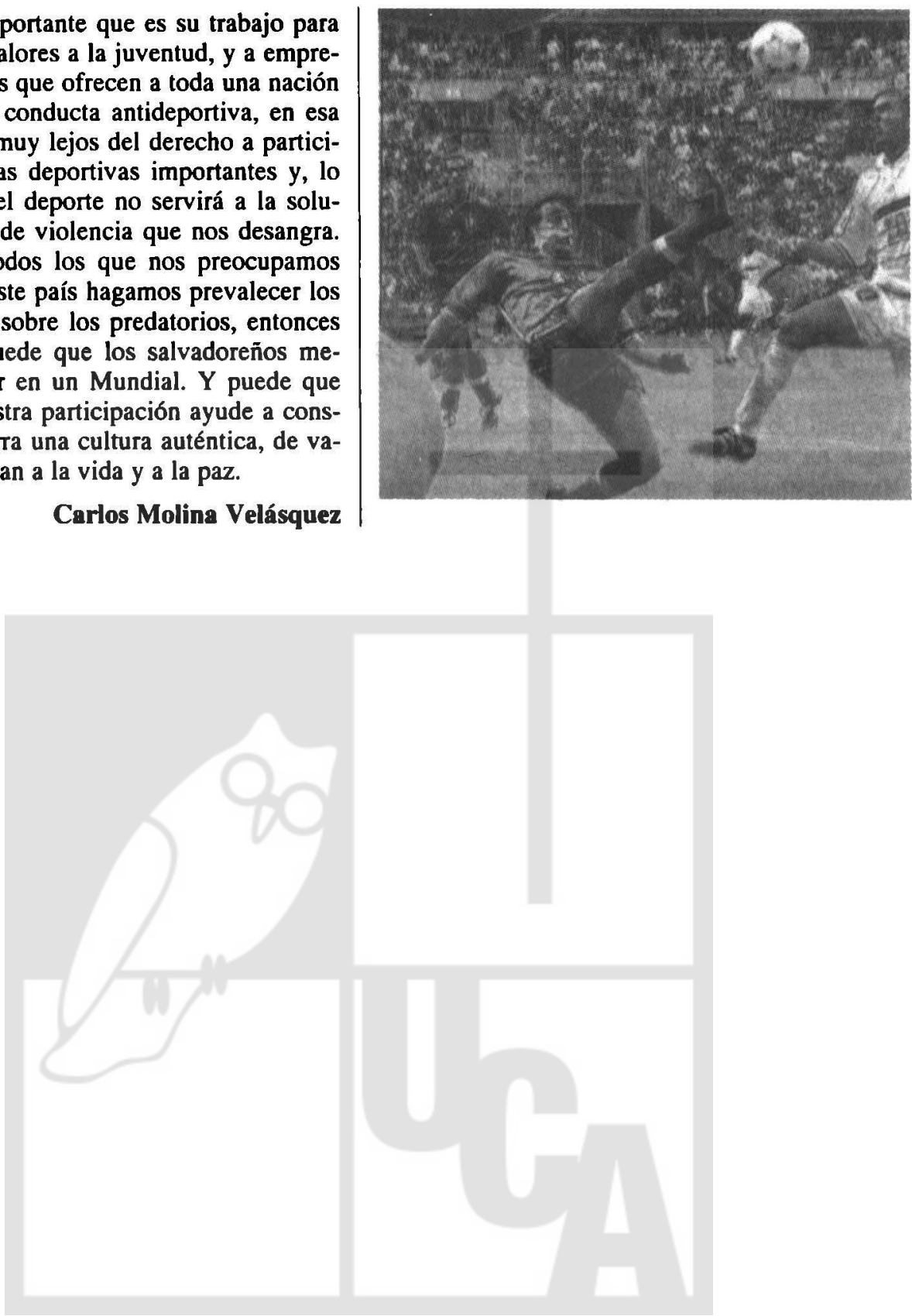